\title{
EXPOSICION BIOLÓGICA A PATÓGENOS HEMÁTICOS Y TEMPORALIDAD LABORAL
}

\author{
BRÍGIDO PÉREZ BERMÚDEZ *, \\ ANA BELÉN NIETO SÁNCHEZ **
}

(*) Médico de Trabajo

(**) Enfermera de Trabajo

Servicio de Prevención de Riesgos Laborales del Área 2.

Hospital Universitario de la Princesa de Madrid

\section{RESUMEN}

La exposición biológica a patógenos hemáticos (E.B.P.H.) y especialmente el pinchazo es el accidente más frecuente en el ámbito sanitario.

Objetivos: El objetivo del estudio es comprobar la mayor siniestralidad en este tipo de accidentes entre los trabajadores eventuales y analizar si existe un perfil determinado.

Metodología: Se analizaron todos los accidentes con riesgo biológico ocurridos durante un año en el Hospital Universitario de la Princesa de Madrid. Se construyó un modelo de regresión logística cuya variable dependiente es el tipo de contrato (eventual o plaza en propiedad), las variables independientes estudiadas fueron: edad, sexo, categoría, lugar del accidente, antigüedad en el puesto, anatomía lesionada, agente que lo produce, turno de trabajo, forma en el que se produjo, mes y día de accidente.

Resultado: Existe un riesgo relativo de 1.96 $(1.48-2.60)$ de producirse un E.B.P.H.en un "eventual". En el análisis multivariante no se observan diferencias significativas entre ambos tipos de trabajadores, excepto el aumento de E.B.P.H. ocurridos entre los trabajadores de plantilla en el mes de Septiembre.

Conclusiones: Aunque existe casi dos veces más riesgo de que exista un accidente biológico entre los eventuales, no existen diferencias significativas en las variables estudiadas en los dos tipos de trabajadores.

\section{PALABRAS CLAVES}

Exposición biológica ocupacional, pinchazo accidenta, siniestralidad y temporalidad en sanidad.

\section{ABSTRACT}

Blood pathogens biological exposure (B.P.B.O) and specially needle stick injury is the most common accident in the sanitary field.

Objectives: The main objective of this research is to check the level of sinestry in this kind of accidents among temporary workers and analyse if there is a certain profile.

Methodology: All accidents with biologic risks happened in a year were analised in the University Hospital "La Princesa" . A logistic regression was performed where the dependent variables to study were: age, sex, grade, place of the accident, seniority, injured body part, producing agent, working shift, way in which it's caused, month and day of the injury.

Results: There is a relative risk of 1.96 (1.48.2.60) higher to happen an B.P.B.O in a temporary worker. Over the other variables studied in the multivariable analysis, no significant differences are detected, but an increase of B.P.B.O among workers in September.

Conclusions: Although there is almost twice the risk of a biological accident among temporary workers, there are no significant diferences in the variables studied over the both kind of workers.

\section{KEY WORDS}

Occupacional Biological Exposition ( OBE ), needle stick injury, siniestry and eventuality in Health system. 


\section{INTRODUCCIÓN}

La siniestralidad debido a la exposición biológica a patógenos hemáticos (E.B.P.H.) y especialmente el pinchazo accidental, es la causa más frecuente e importante en el ámbito sanitario, tanto por las consecuencias para el trabajador (posible infección, ansiedad, estrés, etc.) como por las repercusiones económicas que llevan consigo ${ }^{1,2,3,4,5}$. En numerosos artículos publicados $^{6,7}$ se observa como uno de los factores de riesgo asociados a la exposición accidental, es la inexperiencia de los trabajadores en los procedimientos y en las técnicas sanitarias que requieren manipulación de objetos punzantes. En la sanidad pública española existe en el ámbito laboral un porcentaje elevado de trabajadores (en algunos centros hasta un 50\%) que no ostentan plaza en propiedad, son trabajadores con una movilidad por puesto de trabajo muy alta y en muchos casos, aún llevando un dilatado tiempo de experiencia profesional, se ven sometidos a iniciar aprendizajes continuos al ocupar nuevos puestos de trabajo. El objetivo de este estudio, es contestar a la pregunta de si los trabajadores con una relación contractual con la empresa, que denominamos "eventuales" (interinos, suplentes, médicos y enfermeras en formación, etc.) padecen más E.B.P.H. que los "fijos" y que factores podrían estar relacionados en este tipo de accidente.

\section{MATERIAL Y MÉTODOS}

Estudio de incidencia. Se analizaron todos los accidentes con riesgo biológico, ocurridos en el periodo de Junio de 2005 a Junio de 2006 en el Hospital Universitario de la Princesa, de los 2300 trabajadores que constituyen la plantilla, aproximadamente un $50 \%$ eran eventuales. Se comparan las tasas de siniestralidad por E.B.P.H. entre los eventuales y fijos. Se estudian las variables edad, sexo, categoría, lugar del accidente, antigüedad en el puesto de trabajo, anatomía lesionada, agente que lo produce, turno de trabajo en que se accidenta el trabajador, forma en el que se produjo, mes y día de accidente y la situación contractual del trabajador con la empresa.

Para la comparación de proporciones se utilizó el ji cuadrado y para la comparación de medias la t-student. Para evitar variables confusoras se construyó un modelo de regresión logística no condicional cuya medida de asociación es la odds ratio con sus intervalos de confianza al 95\%. Como variable dependiente se analizó el tipo de contrato (eventual o trabajador con plaza en propiedad).

\section{RESULTADOS}

Durante el periodo a estudio (Junio de 2005 a Junio de 2006) se produjeron un total de 201 exposiciones biológicas a patógenos hemáticos de los que 121 (60 \%) se produjeron en trabajadores con contratos eventuales y 80 (40 \%) tenían plaza en propiedad. La incidencia entre los eventuales es de 12.96 por cada 100 trabajadores, mientras que en los fijos es de 6.20 por cada 100 trabajadores, estas diferencias de incidencias son significativas $(p=0.000)$, existiendo un R.R. de 1.96 (1.48-2.60) veces más de producirse un E.B.P.H. en un "Eventual" que en un "Fijo".

El estudio descriptivo de las variables y bivariante, son expuestas en la tabla I.

Puede observarse, en el análisis bivariante que las variables lugar, categoría, edad y antigüedad en el puesto de trabajo son significativas.

Como es de esperar, tanto la antigüedad como la edad son variables con diferencias significativas entre los dos tipos de trabajadores (tienen menos antigüedad laboral y son más jóvenes los eventuales). En la variable categoría, este tipo de accidente es más frecuente entre los Médicos y "no Sanitarios" eventuales, (fundamentalmente Médicos Internos Residentes y trabajadores de la limpieza). En el resto de las categorías, es más frecuente el accidente entre los trabajadores con plaza en propiedad.

En cuanto a la variable lugar, Quirófano, Reanimación y Anestesia (R.E.A.), U.C.I y Laboratorios, es más frecuente el accidente entre los eventuales.

En el análisis descriptivo temporal, (reflejado en los gráficos 1 y 2) llama la atención, el aumento de los accidentes en el mes de Septiembre, de los trabajadores con plaza en propiedad.

El pinchazo, sigue siendo la forma más frecuente en los dos tipos de trabajadores y el agente que los produce con mayor prevalencia son las agujas. Las suturas son las más importantes entre los eventuales, ya que los M.I.R. son los más involucrados en la técnica de suturar.

La anatomía más implicada son los dedos y las manos y el turno donde más se producen los E.B.P.H. es la mañana. 
Tabla I. Descriptivo de las variables estudiadas.

\begin{tabular}{|c|c|c|c|}
\hline VARIABLES & EVENTUALES & FIJOS & $\mathbf{P}$ \\
\hline EDAD Media (Desviación típica) en años & $30.38(9.02)$ & $41.23(12.89)$ & 0.000 \\
\hline ANTIGÜEDAD Media (Desviac. típica) en años & $5.22(7.30)$ & $17.38(11.75)$ & 0.000 \\
\hline SEXO & & & 0.785 \\
\hline Mujeres & $75.8 \%$ & $77.5 \%$ & \\
\hline CATEGORIAS & & & 0.005 \\
\hline Medico & $45.8 \%$ & $32.5 \%$ & \\
\hline Enfermería & $30.0 \%$ & $51.3 \%$ & \\
\hline Aux. Enfermería & $7.5 \%$ & $11.3 \%$ & \\
\hline Técnico Especialista & $3.3 \%$ & $2.5 \%$ & \\
\hline No sanitarios & $13.3 \%$ & $2.5 \%$ & \\
\hline LUGAR & & & 0.050 \\
\hline Habitación paciente -Control-Pasillo & $23.3 \%$ & $33.8 \%$ & \\
\hline Quirófano & $43.3 \%$ & $37.5 \%$ & \\
\hline REA-UCI-Dialisis-Endoscopias & $11.7 \%$ & $6.3 \%$ & \\
\hline Urgencias & $6.7 \%$ & $2.5 \%$ & \\
\hline Consultas & $2.5 \%$ & $10.0 \%$ & \\
\hline Laboratorios-Radiologia & $11.7 \%$ & $7.5 \%$ & \\
\hline Otros & $0.8 \%$ & $2.5 \%$ & \\
\hline FORMA & & & 0.774 \\
\hline Pinchazo & $75.5 \%$ & $78.8 \%$ & \\
\hline Sapicadura & $17.5 \%$ & $13.8 \%$ & \\
\hline Otros EBO & $7.5 \%$ & $7.5 \%$ & \\
\hline AGENTE & & & 0.701 \\
\hline Aguja & $45.0 \%$ & $51.3 \%$ & \\
\hline Sutura & $20.0 \%$ & $12.5 \%$ & \\
\hline Bisturí & $3.3 \%$ & $2.5 \%$ & \\
\hline Otros objetos punzantes & $13.3 \%$ & $12.5 \%$ & \\
\hline Sangre-Secreciones & $18.3 \%$ & $21.2 \%$ & \\
\hline ANATOMIA AFECTADA & & & 0.359 \\
\hline Dedos & $74.2 \%$ & $75.0 \%$ & \\
\hline Manos & $5.8 \%$ & $10.0 \%$ & \\
\hline Cabeza & $17.5 \%$ & $13.8 \%$ & \\
\hline Cadera-Piernas-brazos & $1.7 \%$ & $1.3 \%$ & \\
\hline TURNOS & & & 0.456 \\
\hline Mañana & $75.8 \%$ & $77.5 \%$ & \\
\hline Tarde & $12.5 \%$ & $7.5 \%$ & \\
\hline Noche-Guardias & $11.7 \%$ & $15.0 \%$ & \\
\hline
\end{tabular}


Respecto a la distribución temporal de los accidentes, atendiendo a los meses del año (gráfico 1), vemos que existen dos picos en las tasas (accidentes/1000) uno que se produce en otoño y otro en pri- mavera y curiosamente en el mes de septiembre, se observa excepcionalmente un aumento de E.B.P.H., entre los trabajadores fijos, como puede apreciarse.

Gráfico 1. Distribución de las tasas de exposición durante el año de estudio.

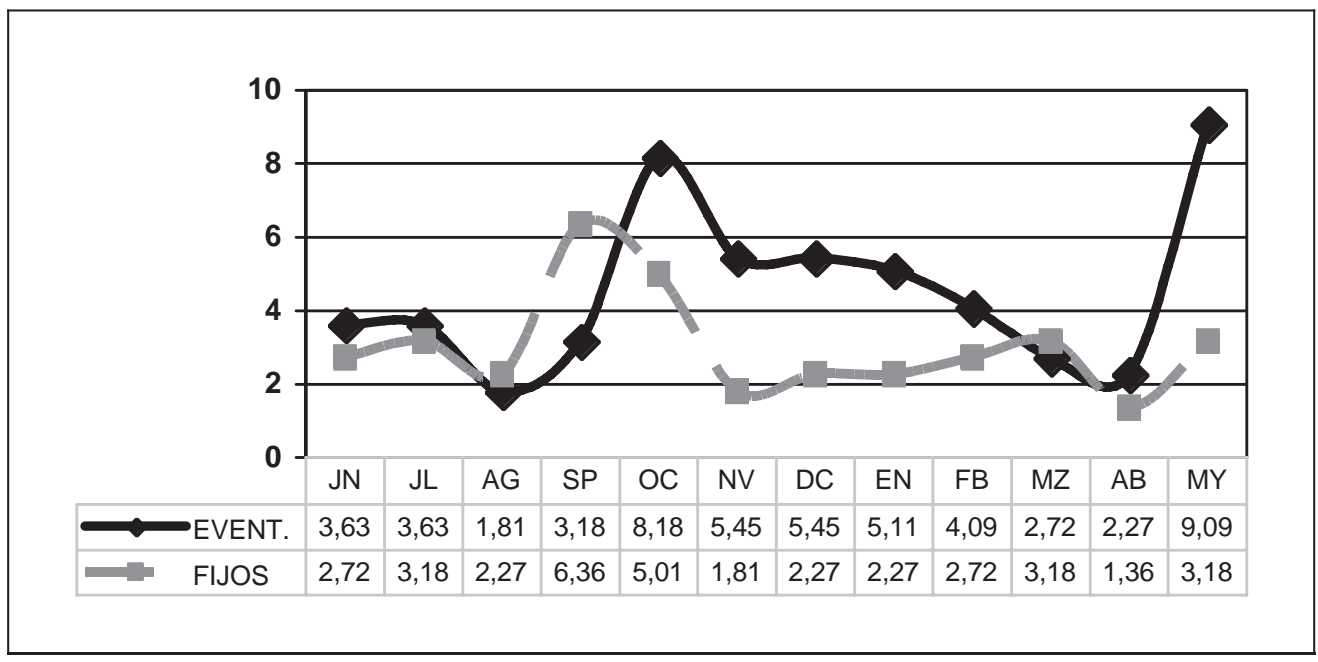

En el gráfico 2, refleja el comportamiento de las tasas de exposiciones, respecto a los días de la semana.
Por último, realizamos un modelo de regresión logística, cuyos resultados se exponen en la tabla II.

Gráfico 2. Distribución de las tasas de exposición durante los días de la semana.

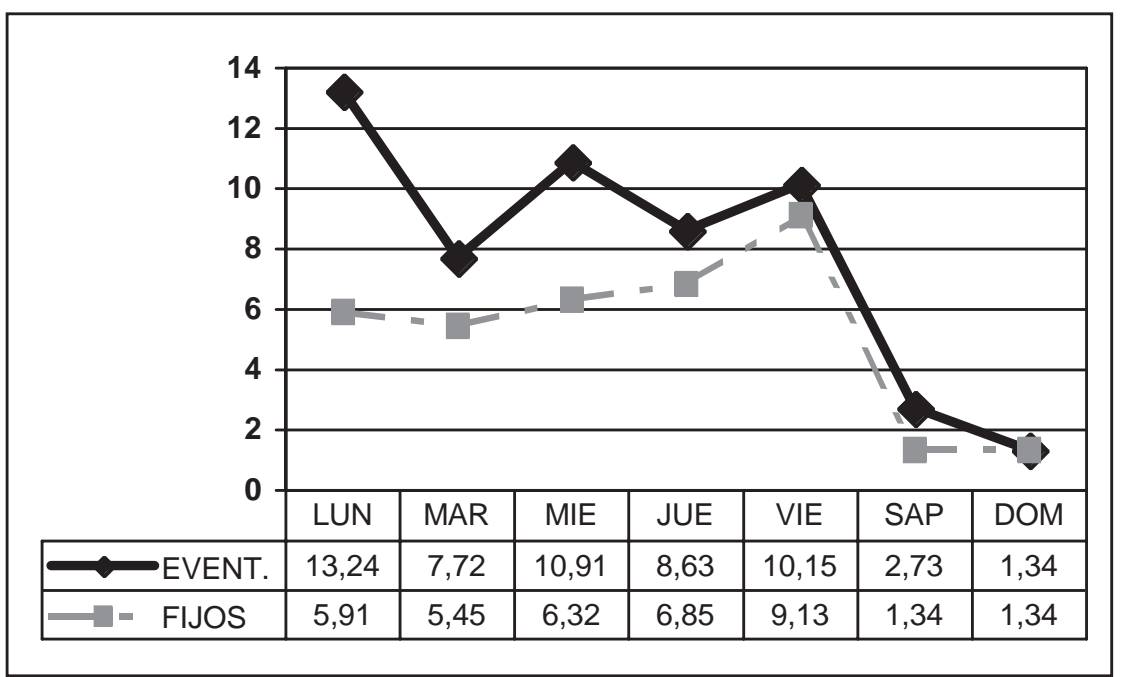


Tabla II. Resultado de la regresión logística

\begin{tabular}{|c|c|c|}
\hline VARIABLE & OR & IC 95\% \\
\hline SEXO & 1.71 & $0.70-4.16$ \\
\hline \multicolumn{3}{|l|}{ MES } \\
\hline Enero & 1 & \\
\hline Febrero & 1.49 & $0.28-7.80$ \\
\hline Marzo & 2.04 & $0.29-13.98$ \\
\hline Abril & 0.69 & $0.07-6.73$ \\
\hline Mayo & 1.02 & $0.22-4.70$ \\
\hline Junio & 2.22 & $0.40-12.31$ \\
\hline Julio & 1.34 & $0.24-7.31$ \\
\hline Agosto & 4.12 & $0.57-29.69$ \\
\hline Septiembre & 4.40 & $0.98-21.75$ \\
\hline Octubre & 1.34 & $0.30-5.84$ \\
\hline Noviembre & 0.68 & $0.12-3.79$ \\
\hline Diciembre & 1.16 & $0.20-6.66$ \\
\hline \multicolumn{3}{|l|}{ LUGAR DEL ACCIDENTE } \\
\hline Habitación del paciente -Control-Pasillos & 1 & \\
\hline Quirófanos & 0.29 & $0.01-8.13$ \\
\hline REA-UCI-Diálisis & 0.17 & $0.01-5.01$ \\
\hline Urgencia-U.D.H. & 0.10 & $0.01-3.63$ \\
\hline Consultas & 0.06 & $0.01-3.13$ \\
\hline Laboratorios-Rx-Anat.Pat & 0.50 & 0.01-18.87 \\
\hline Otros & 0.18 & $0.01-6.43$ \\
\hline \multicolumn{3}{|l|}{ FORMA EN QUE SE PRODUCE } \\
\hline Pinchazo & 1 & \\
\hline Salpicadura & 0.54 & $0.09-3.08$ \\
\hline otras & 1.89 & $0.33-10.69$ \\
\hline \multicolumn{3}{|l|}{ CATEGORIAS } \\
\hline Médicos & 1 & \\
\hline Enfermería & 2.25 & $0.75-6.73$ \\
\hline Aux. Enfermería & 2.76 & $0.63-12.05$ \\
\hline Técnico & 0.98 & $0.08-11.69$ \\
\hline No Sanitarios (Limpieza, celadores, etc) & 0.21 & 0.03-1.06 \\
\hline \multicolumn{3}{|l|}{ AGENTE QUE LO PRODUCE } \\
\hline Aguja & 1 & \\
\hline Sutura & 1.02 & $0.34-3.02$ \\
\hline Bisturí & 0.83 & $0.09-7.66$ \\
\hline Otros objetos punzantes & 0.72 & $0.23-2.23$ \\
\hline Sangre-secreciones & 1.46 & $0.26-8.04$ \\
\hline \multicolumn{3}{|l|}{ TURNOS } \\
\hline Mañana & 1 & \\
\hline Tarde & 0.67 & $0.20-2.28$ \\
\hline Noche-Guardia & 0.92 & $0.32-2.61$ \\
\hline
\end{tabular}


Prueba de Hosmer-Lemeshow $\mathrm{p}=0.83$

El área bajo la curva R.O.C. es de 0.78 (I.C al $95 \%$ de 0.71 - 0.85).

En la construcción del modelo no se han introducido las variables edad y antigüedad por la alta colinealidad.

\section{DISCUSIÓN}

Se confirma que los E.B.P.H., son casi 2 veces más frecuentes entre los eventuales que entre los fijos. Es lógico pensar, que este hecho se produce como consecuencia de que el trabajador eventual es menos experto en los procedimientos.

En general, como puede observarse en el análisis multivariante, no existen diferencias significativas entre los dos tipos de trabajadores en las variables estudiadas.

En la variable "Categorías" los trabajadores agrupados como "no sanitarios" existe una OR de 4, 76 (1/0.21) más de padecer un E.B.P.H. en los eventuales que en los fijos, esto es fundamentalmente debido a que en esta agrupación existe una prevalencia alta de trabajadores de la limpieza, que en alta proporción son eventuales de la empresa subcontratada. En numerosas ocasiones, los accidentes se han producido al manipular los residuos que llevaban objetos punzantes desechados. Debemos hacer un esfuerzo, en informar a estos trabajadores sobre el riesgo que supone manipular estos residuos en centros sanitarios, así como incidir en la importancia de clasificar los residuos correctamente.

En cuanto a la variable mes en el que se produce el accidente, llama la atención, el aumento significativo entre los trabajadores fijos, durante septiembre, mes de regreso al trabajo tras el descanso vacacional, produciéndose una O.R. 4.40 veces más entre los fijos que entre los eventuales. No encontramos una clara explicación, pero podría deberse, a un efecto de cierta pérdida en las habilidades de los procedimientos tras el descanso vacacional, sumado al aumento de actividad; no obstante para un mejor conocimiento de este hallazgo, deberíamos comprobar en años sucesivos si esto es así. También es llamativo, los dos picos de siniestralidad que se producen en primavera y en otoño, ya recogidos en otros trabajos $^{6,7}$, posiblemente relacionado con la mayor actividad en estos periodos.

Respecto al comportamiento de los E.B.P.H. durante la semana de lunes a viernes, se observa más siniestralidad en los eventuales y sólo los viernes, los trabajadores fijos se igualan en tasa de siniestralidad a los eventuales.

En cuanto al lugar, sin ser significativo, se observa el alto porcentaje de E.B.P.H. que se producen en la habitación del paciente, pasillo o control, en muchas ocasiones a consecuencias del hecho de deshacerse del material usado, siendo a la vez este hecho más frecuente en el trabajador fijo, quizás por un exceso de confianza.

En la forma en que se produce, es interesante observar que casi un $20 \%$ de los E.B.P.H. tienen relación con la salpicadura. Es importante que los trabajadores sean conscientes de la posibilidad que esto ocurra y eliminar o disminuir los efectos con los equipos de protección individual correspondientes (gafas, mascarillas, etc.).

Los agentes más frecuentemente involucrados son las agujas, pero la maniobra de suturar es muy prevalente entre los eventuales, en numerosas ocasiones debido al aprendizaje de los MIR en técnicas quirúrgicas, como ya hemos comentado.

Los turnos tienen un comportamiento parecido, siendo lógicamente el turno de la mañana en el que más siniestralidad se produce, debido a ser el turno donde la actividad es mayor.

En resumen, debemos indicar, que el perfil de los factores asociados a los E.B.P.H. es similar entre los dos tipos de trabajadores a estudio (eventuales y fijos) y que la diferencia sustancial, es la "inexperiencia" en las técnicas a desarrollar en los diferentes puestos de trabajo. Por tanto, consideramos como objetivo clave para disminuir este tipo de siniestralidad, una mejor preparación, dedicación y formación al trabajador que se incorpore a un nuevo puesto de trabajo, y esta formación debe ser un compromiso de todos, compañeros, supervisores, jefes y responsables. 


\section{BIBLIOGRAFÍA}

1. Campins Martí M, Hernández Navarrete MJ, editores, y Grupo de Trabajo EPINETAC (Sociedad Española de Medicina Preventiva, Salud Pública e Higiene). Estudio y seguimiento del riesgo biológico en el personal sanitario. Madrid: Grupo de Trabajo EPINETAC; 2002.

2. Comisión Central de Salud Laboral y Grupo Español de Registro de Accidentes Biológicos en Trabajadores de Atención de Salud. Accidentes biológicos en profesionales sanitarios. 3.a ed. Madrid: Insalud; 1997

3. Monge V, Mato G, Mariano A, Fernández C, Fereres J, y Grupo GERABTAS. Epidemiology of biological-exposure incidents among spanish healthcare workers. Infect Control Hosp Epidemiol. 2001;22:776-80

4. Solano, Víctor M., Hernandez, María J., Montes, Francisco Javier et al. Actualización del coste de las inoculaciones accidentales en el personal sanitario hospitalario. Gac Sanit, ene.-feb. 2005, vol.19, no.1, p.29-35.

5. Laufer FN, Chiarello LA. Application of costeffectiveness methodology to the consideration of needlestick-prevention technology. Am J Infect Control. 1994;22:75-82

6. Pérez Bermúdez, B., Tenias Burillo, J.M $\mathbf{M}^{\mathrm{a}}$, Tolosa Martinez, N. et al. Accidentes de trabajo en un hospital de agudos. Rev. Esp. Salud Publica, Mar./Abr. 1998, vol.72, no.2, p.127-136. ISSN 1135-5727.
7. Gallardo Lopez, Ma Teresa, Masa Calles, Josefa, Fernández-Creuet Navajas,, Rafael et al. Factores asociados a los accidentes por exposición percutánea en personal de enfermería en un hospital de tercer nivel. Rev. Esp. Salud Publica, Jul./Ago. 1997, vol.71, no.4, p.369-381.

8. Blázquez R, Moreno S, Menasalvas A, Guerrero C, Novoa A, Segovia M. Exposición ocupacional a patógenos hemáticos en trabajadores sanitarios. Enferm Infecc Microbiol Clin 2001;19:156-60.

9. De Juanes JR. Problemática de los accidentes con sangre y/o derivados en un hospital general. Cinco años de seguimiento. Rev Esp Microbiol Clin 1986;1(5): 317-21.

10. Gerberding JL. Occupational exposure to HIV in Health Care Settings. N Engl J Med 2003; 348(9):826-33. 23. Baldo V, Floreani A, Dal Vecchio L, Cristofoletti M, Carletti M, Majori S, et al. Occupational Risk of Blood-Borne Viruses in Healthcare Workers: A 5-Year Surveillance Program. Infect Control Hosp Epidemiol 2002;23(6): 325-7.

11. De Andrés R, Pérez L, Contreras G, Nájera R, and the European Collaborative Study Group on Accidental Exposure to HIV. Update on European Union multicenter study of occupational exposure to HIV in health care workers. En: XI International Conference on AIDS; 1996 Jul; Vancouver 